\section{U Leiden University MC Medical Center}

M. de Fouw ${ }^{1}$, Y. Stroeken ${ }^{1}$, B. Niwagaba ${ }^{2}$, M. Musheshe ${ }^{2}$, J. Tusiime ${ }^{2}$, I. Sadayo ${ }^{2}$,

R. Reis ${ }^{3}$, A.A.W. Peters ${ }^{1}$ and J.J.Beltman ${ }^{1}$ ${ }^{1}$ Dept of Gynaecology, Leiden University Medical Center ${ }^{2}$ Dept of Technologies for Rural transformation, African Rural University, Uganda

${ }^{3}$ Dept of Public Health and Primary care, Leiden University Medical Center, Dept of Anthropology University of Amsterdam

\title{
Involving men in cervical cancer prevention; a qualitative enquiry into male perspectives on screening and HPV-vaccination in Mid-Western Uganda
}

\section{Introduction}

Evidence-based preventive strategies for cervical cancer in low-resource setting have been developed, but implementation is challenged and uptake remains low.

Women and girls experience barriers to attend screening and human papilloma virus (HPV) vaccination programs. Male support has been proven successful in uptake of other reproductive healthcare services in Uganda.

This qualitative study aimed to understand the perspectives of males on cervical cancer screening and HPV vaccination in Uganda.

\section{Results}

Men were willing to support their wives for screening and their daughters for HPV-vaccination. Men can discuss reproductive health issues with their wives, but only mothers or close female relatives can discuss directly with their daughters.

"A man can never enter into such discussions." (FGD 4)

Misperceptions, such as family planning and poor personal hygiene thought to cause cervical cancer, and misperception of the preventative aspect of screening and vaccination were common.

"If a woman has this cancer and she urinates somewher and another woman also urinates on that same place,

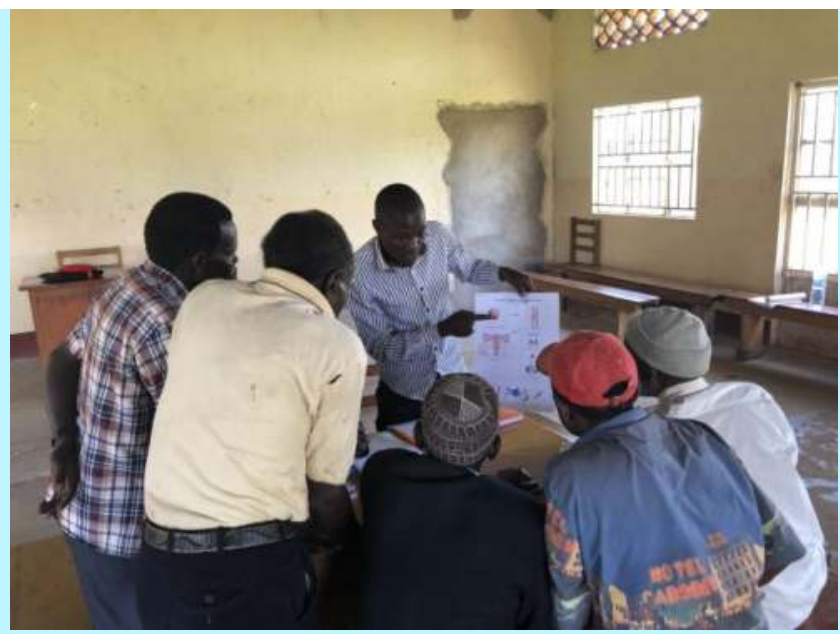

Moderator provides information on HPV vaccination

\section{References}

- Hasahya OT, Berggren V, Sematimba D, Nabirye RC, Kumakech E. Beliefs, perceptions and health-seeking behaviours in relation to cervical cancer: a qualitative study among women in U
completion of an $H P V$ vaccination campaign. Glob Health Action. 2016;;: 29336 .

\section{- Lim IN Ojp A Ca}

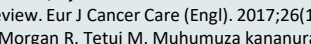

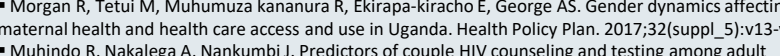
- Muhindo R, Nakalega A, Nankumbij. Predictors of couple HIV counseling and testing among ad
residents of Bukomero sub-county Kiboga district, rural Uganda. BMC Public Health. 2015;15:117. - Mukama $T$, Ndejij R, Musabbyimana A, Halage AA, Musoke D. Women's's knowledge and attitudes towards
cervical cancer prevention: a cross sectional study in Eastern Uganda. BMC Womens Health. 2017;17(1):9. cervical cancer prevention: a cross sectional study in Eastern Uganda. BNC Womens
- Mutyaba T, Mirembe $F$, Sandin $S$, Weiderpass E. Male partner involvement in reducic
after

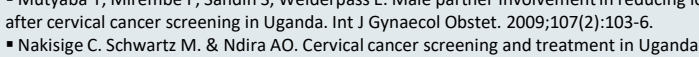

\section{Methods}

Focus group discussions were conducted by two local male moderators with men aged 25 to 60 years, who were married and/or had daughters, in Kagadi district, MidWestern Uganda.

All interviews were transcribed verbatim and thematically analyzed using an inductive approach

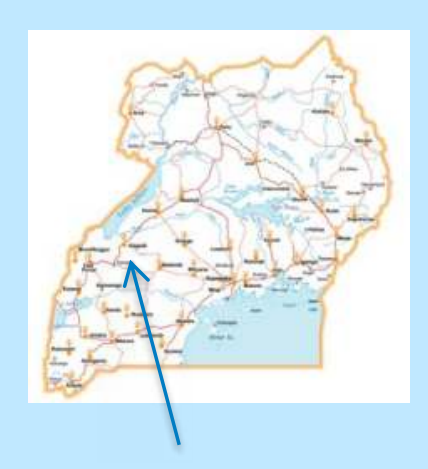

Kagadi district
"Others say that the plan is that doctors want to vaccinate
our girls, daughters and end their productivity." (FGD 10)

Women with cervical cancer suffer from stigmatization and family problems due to loss of fertility, less marital sexual activity, domestic violence and decreased economic productivity.

\section{"This infection is through sexual intercourse so the man will} know that his wife cheated on him, that is why she has cervical cancer (..) Now the man will start doubting
and he may chase her from his home." (FGD 10) "A woman without uterus is no longer a woman." (FGD 3)
"Cancer scares men more than HIV/AIDS. AIDS has tablets,
but with cancer, where are they?" (FGD 3) but with cancer, where are they?" (FGD 3)

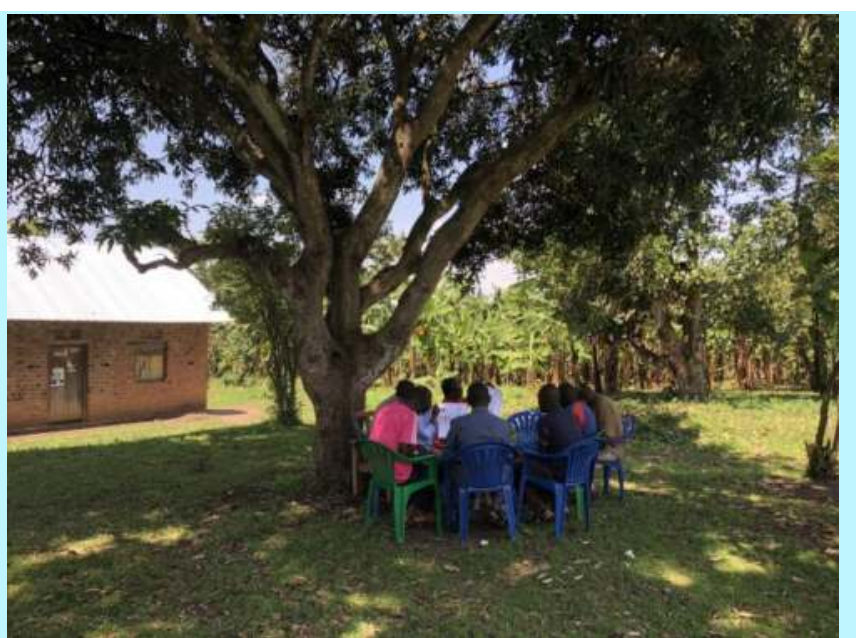

A focus group discussion in a secluded public space

\section{Results - Participants}

In total eleven FGDs of approximately 1.5 hours each were conducted with 67 men.

After nine FGDs saturation of themes was achieved. Two more FGDs were conducted to confirm saturation of themes and execute in-depth exploration of some subthemes.

Information about the participants: - all were literate, all were married

-93\% (62/67) completed primary or secondary education;

$4 \%(3 / 67)$ had a relative affected by cervical cancer;

- $20 \%(11 / 56)$ reported daughters were vaccinated;

- 19\% (13/67) reported wives were screened;

-96\% (64/67) would accept screening for their wives.

\section{Conclusions}

Ugandan men were willing to support cervical cancer prevention for their wives and daughters.

Men perceived cervical cancer as a serious and potentially fatal disease, which can be a motivation to participate in prevention programs.

Limited knowledge among men about the causes of cervical cancer, the preventative aspect and target groups for HPV-vaccination and screening can limit uptake of both services.

Screening and vaccination programs should actively involve men in awareness to increase uptake and acceptance of the programs.

\section{Future perspectives}

We recommend that:

1. awareness programs should address the importance of prevention for all women and girls.

2. Awareness programs should invest in thorough health education for both women and men. In rura Uganda, radio messages are effective in reaching men. Male group conversations could provide a better opportunity to achieve behavioral change.

3. Health education should include information about preventive measures for men, e.g. condom use, male circumcision, and support screening and vaccination.

\section{Acknowledgements}

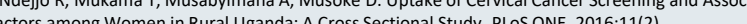
- Ndejjo R, Mukama T, Kiguli J, Musoke D. Knowledge, facilitators and barriers to cervical cancer screening - Strategic plan for cervical a cancer prevention and control in I Uganda (2010-2014).

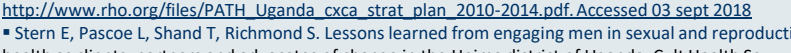

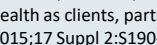
- Turiho AK, Okello ES, Muhwezi WW, Katathoire AR. Perceptions of human papillomavirus vaccination of dolescent schoolgiris in western Uganda and thei ing - Twinomujuni C, Nuwaha F, Babirye JN. Understanding the Low Level of Cervical Cancer Screening in Twinomujunic, Nuwaha F, Babirye IN. Understanding the Low Level of Cervical Cancer S
Masaka Uganda Using the ASE Model: A Community-Based Survey. PLoS ONE. 2015;10(6).
The authors would like to thank all men that participated in the focus groups and shared their opinions and beliefs with us.

Our thanks and appreciation goes out to the staff of the Ugandan Rural Development and Training Program, in particular the screening team, and African Rural University for their support for this research project. 